\title{
Evaluación hemodinámica en escenarios con recursos limitados
}

\author{
Sergio Edgar Zamora-Gómez ${ }^{1}$, Marco Antonio Toledo-Rivera², Luis Antonio Gorordo-Delso/*, \\ Marcos Antonio Amezcua-Gutiérrez ${ }^{3}$, José Obeth Montoya-Rojo ${ }^{4}$ e Iván Mauricio Lima-Lucero ${ }^{5}$
}

${ }^{1}$ Unidad de Cuidados Intensivos, Hospital de Beneficencia Española de Tampico, Tamaulipas; ${ }^{2}$ Unidad de Cuidados Intensivos, Hospital Sedna, Ciudad de México; ${ }^{3}$ Unidad de Cuidados Intensivos Adultos, Hospital Juárez de México, Ciudad de México; ${ }^{4}$ Unidad de Cuidados Intensivos, Hospital Regional de Especialidades 30, IMSS, Mexicali, Baja California; ${ }^{5}$ Unidad de Terapia Intensiva Cardiovascular, Instituto Nacional de Cardiología "Ignacio Chávez", Ciudad de México. México

\begin{abstract}
Resumen
El monitoreo hemodinámico es una de las piedras angulares de la atención en los Servicios de Urgencias y Unidades de Cuidados Intensivos; sin embargo, muchos hospitales operan con recursos limitados. Esta heterogeneidad es habitual en países de todos los niveles socioeconómicos, por ello el médico debe aprender las bases fisiológicas y los métodos simples, de los que se dispone en la mayoría de los entornos, y evaluar las ventanas clínicas y paraclínicas de forma sistematizada para detectar e intervenir de forma eficaz en pro de la salud del paciente. Este trabajo sintetiza tres aspectos clínicos: el análisis de la captación, traslado, entrega y consumo de oxígeno y cómo modificar estos pasos de forma sencilla con el soporte gasométrico y ecográfico.
\end{abstract}

Palabras clave: Monitoreo. Recursos de salud. Ácido láctico. Perfusión.

\section{Hemodynamic evaluation in resource-limited settings}

\begin{abstract}
Hemodynamic monitoring is one of the cornerstones of care in the Emergency Room and Intensive Care Units; however, many hospitals operate with limited resources. This heterogeneity is common in countries of all socioeconomic levels, so the physician must learn the physiological bases and the simple methods, which are available in most environments, evaluating the clinical and paraclinical windows in a systematized way to detect and intervene in an effective way in favor of the patient's health. This work synthesizes three clinical aspects, the analysis of the uptake, transfer, delivery and consumption of oxygen and how to modify these steps in a simple way with gasometric and ultrasound support.
\end{abstract}

Key words: Hemodynamic monitoring. Health resources. Lactic acid. Perfusion.

\section{Correspondencia:}

*Luis Antonio Gorordo-Delsol

E-mail: luis.@gorordodelsol@icloud.com
Fecha de recepción: 05-04-2019

Fecha de aceptación: 18-09-2019 DOI: 10.24875/REIE.19000028
Disponible en internet: 06-11-2019 Rev Educ Investig Emer. 2019;1(3):106-112 www.medicinadeemergencias.com

604-6520 () 2019 Sociedad Mexicana de Medicina de Emergencias, AC. Publicado por Permanyer México SA de CV. Este es un artículo open access bajo la licencia CC BY-NC-ND (http://creativecommons.org/licenses/by-nc-nd/4.0/). 


\section{Introducción}

En los escenarios críticos es vital detectar al paciente que se encuentra en choque, es decir, hipoperfusión generalizada, y a aquel que está en alto riesgo de deterioro, así como tener herramientas para soportar las decisiones terapéuticas y evaluar la respuesta del paciente, con el objetivo de brindar el tratamiento inmediato y disminuir las complicaciones asociadas a la reanimación. Un gran problema, que se observa con frecuencia en Latinoamérica, es la heterogeneidad de los recursos tecnológicos disponibles ${ }^{1}$, lo que supone un reto para el médico. Es complejo determinar qué es un escenario con recursos limitados y cuáles son las herramientas con las que se cuenta, algunos autores consideran que los gasómetros o el ultrasonido no son parte de estos entornos, sin embargo, con la reducción de costos y el aumento de la portabilidad de los equipos, estas dos herramientas llegan a más espacios.

\section{Prioridades de identificación}

Establecer prioridades de identificación del estado hemodinámico permite actuar de manera pronta y eficaz, así como evitar acciones innecesarias o deletéreas para el paciente. Consisten en identificar al paciente en riesgo de o con hipoperfusión, y establecer el perfil hemodinámico subyacente que afecta la perfusión tisular. Podemos agrupar las herramientas en variables clínicas y marcadores bioquímicos, sumado la ultrasonografía crítica, que adquiere relevancia por la relación costo-efectividad, disponibilidad e inocuidad.

\section{Ventanas clínicas de perfusión}

El consenso internacional de choque recomienda el uso de «ventanas» clínicas para evaluar la perfusión tisular:

- Neurológica. Se sospecha hipoperfusión cuando el paciente presenta disminución del estado de consciencia, agitación o incluso agresividad. Sin embargo, esta ventana ve disminuida su efectividad para evaluar la perfusión tisular cuando el paciente presenta patología neurológica aguda, secuelas de eventos previos o sedación.

- Renal. Si el sujeto presenta flujo urinario $\geq 0.5 \mathrm{ml} / \mathrm{kg} / \mathrm{h}$, la perfusión tisular está conservada, sin embargo, se necesitan al menos 6 horas de acuerdo con las clasificaciones actuales para establecer lesión renal aguda, por lo que no es efectiva para la evaluación inicial; además de que los egresos hídricos contemplados por medio de la uresis deben correlacionarse con los ingresos, de manera que el valor absoluto de uresis no es tan relevante como lo es la capacidad del riñón de producir orina de acuerdo al aporte hídrico recibido. No es valorable en pacientes oligúricos o anúricos crónicos.

- Cutánea. La redistribución de flujo durante hipoperfusión condiciona cambios cutáneos caracterizados por parches marmóreos, que de acuerdo con su extensión a partir de la rodilla se han relacionado con riesgo de mortalidad, clasificación conocida como «puntaje de moteado»; pero la exposición al frío, afectación cutánea por enfermedades autoinmunes, quemaduras o incluso las variedades de pigmentación de la piel pueden sesgar la evaluación de esta ventana ${ }^{3}$.

\section{Índice de choque}

El índice de choque (IS) es la integración de dos variables: frecuencia cardiaca/presión arterial sistólica. Se utiliza en la evaluación de pacientes con choque hemorrágico y está asociado con alteración de la perfusión tisular y malos desenlaces cuando el valor es $\geq 0.8^{4}$. La alteración del IS debe alertar para corroborar hipoperfusión tisular, aunque existen situaciones en los cuales el IS puede estar normal a pesar de hipoperfusión, como por ejemplo bloqueos en la conducción cardiaca, marcapaso cardiaco, disminución de estimulación adrenérgica por fármacos hipnóticos sedantes 0 lesión medular, por citar algunos ${ }^{5}$.

\section{Marcadores bioquímicos de perfusión: diferencia venoarterial de $\mathrm{CO}_{2}$, ratio $\mathrm{CO}_{2} / \mathrm{O}_{2}$ y lactato}

La diferencia entre el valor de dióxido de carbono $\left(\mathrm{CO}_{2}\right)$ obtenido de una muestra venosa mixta o central y el $\mathrm{CO}_{2}$ obtenido de una muestra arterial correlaciona con incremento del metabolismo anaerobio cuando es $\geq 6 \mathrm{mmHg}^{6}$; este incremento en la diferencia de $\mathrm{CO}_{2}$ venoarterial $\left(\mathrm{DvaCO}_{2}\right)$ se debe al incremento de hidrogeniones en el plasma provenientes del ambiente intracelular como resultado del metabolismo anaerobio; al ingresar al plasma los hidrogeniones son amortiguados y metabolizados a $\mathrm{CO}_{2}^{7}$. De igual manera se ha establecido un punto de corte $\geq 8 \mathrm{mmHg}$ de $\mathrm{DvaCO}_{2}$ cuando la muestra es venosa periférica. El incremento de $\mathrm{DvaCO}_{2}$ puede deberse a gasto cardiaco (GC) inadecuado, disfunción mitocondrial, o ambas ${ }^{8}$.

La ratio entre la $\mathrm{DvaCO}_{2}$ y la diferencia arteriovenosa de $\mathrm{O}_{2}\left(\mathrm{DavO}_{2}\right)$ se correlaciona con hipoperfusión tisular 
cuando es $\geq 1.68 \mathrm{mmHg} / \mathrm{ml}$, con sensibilidad del $90 \%$ y especificidad del $100 \%$ en comparación con el lactato, el cual con un punto de corte $\geq 4.6 \mathrm{mmol} / \mathrm{l}$ obtuvo una sensibilidad del $69 \%$ y una especificidad del $77 \%$. Se calcula con la siguiente fórmula:

$$
\frac{\mathrm{DvaCO}_{2}(\mathrm{mmHg})}{\mathrm{DavO}_{2}\left(\frac{\mathrm{ml}}{\mathrm{min}}\right)}=\frac{\mathrm{CO}_{2} \mathrm{v}-\mathrm{CO}_{2} \mathrm{a}}{\mathrm{CaO}_{2}-\mathrm{CvO}_{2}}
$$

Es importante recalcar que la elevación de niveles de lactato no solo corresponde a hipoperfusión tisular, sino que existen muchas otras causas presentes en el paciente crítico que pueden generar dicha elevación, por lo tanto la recomendación es utilizar la $\mathrm{DvaCO}_{2}$, la ratio $\mathrm{DvaCO}_{2} / \mathrm{DavO}_{2}$ y el lactato para tener mayor sustento y a su vez monitorizar la respuesta al tratamiento, ya que los cambios en la $\mathrm{DvaCO}_{2}$ y la ratio $\mathrm{DvaCO}_{2} / \mathrm{DavO}_{2}$ se manifiestan más rápidamente que la depuración del lactato, sobre todo si el paciente tiene afectación hepática, renal y muscular.

\section{Saturación venosa central y extracción de oxígeno}

El análisis de gases sanguíneos es probablemente la herramienta diagnóstica más usada en áreas críticas. La saturación venosa central de oxígeno $\left(\mathrm{SvO}_{2}\right)$ es una herramienta muy utilizada, sin embargo, para su correcto uso es importante comprender que cualquier cambio en la función pulmonar, hemodinámica, metabólica 0 en el trasporte de oxígeno afectará la $\mathrm{SvO}_{2}{ }^{10}$; en otras palabras, al analizar un valor de $\mathrm{SvO}_{2}$ estamos analizando el resultado de la interacción de todas sus determinantes:

- Ingreso de oxígeno (sistema respiratorio).

- Transporte de oxígeno (hemoglobina).

- Disponibilidad del oxígeno (GC).

- Consumo de oxígeno $\left(\mathrm{VO}_{2}\right)$ (tejidos).

Por lo tanto, la evaluación gasométrica de una muestra venosa central y su relación con la oximetría de pulso o la saturación obtenida de una gasometría arterial proporciona más información que el análisis de un valor gasométrico aislado.

\section{Evaluación de la extracción de oxígeno}

La extracción de oxígeno $\left(\mathrm{O}_{2} \mathrm{ER}\right)$ es la relación entre el $\mathrm{VO}_{2}$ y el aporte de oxígeno $\left(\mathrm{DO}_{2}\right)^{11}$.

$$
\mathrm{O}_{2} \mathrm{ER}=\frac{\mathrm{VO}_{2}}{\mathrm{DO}_{2}}=\frac{\mathrm{SaO}_{2}-\mathrm{SvO}_{2}}{\mathrm{SaO}_{2}}
$$

La $\mathrm{O}_{2} \mathrm{ER}$ representa el porcentaje de oxígeno que la célula extrae de la sangre arterial. El oxígeno que no
Tabla 1. Análisis del comportamiento de la extracción de oxígeno $\left(\mathrm{O}_{2} \mathrm{ER}\right)$ de acuerdo con sus determinantes principales. $\mathrm{DO}_{2}$ obtenida por el gasto cardiaco y $\mathrm{VO}_{2}$ obtenida por el metabolismo celular

\begin{tabular}{|l|l|}
\hline \multicolumn{2}{|c|}{ Extracción de Oxigeno O2ER } \\
\hline$<\mathbf{2 0} \%$ & $>\mathbf{3 0} \%$ \\
\hline $\begin{array}{l}\text { Disponibilidad de oxígeno } \\
\text { Gasto cardiaco }\end{array}$ & Disponibilidad de oxígeno \\
alto (hiperdinamia) & Gasto cardiaco \\
0 & bajo (hipodinamia) \\
Consumo de oxígeno & 0 \\
Metabolismo celular bajo & Consumo de oxígeno \\
(necroapoptosis) & Metabolismo celular alto \\
\end{tabular}

es extraído regresa por la circulación venosa a cavidades derechas del corazón y posteriormente a ser reoxigenada por los pulmones (hematosis).

La $\mathrm{O}_{2} \mathrm{ER}$ normal varía por región, sin embargo de manera global en condiciones de no estrés se considera adecuada para satisfacer las demandas metabólicas del 20 al $30 \%$.

Cuando se evalúa la $\mathrm{O}_{2} \mathrm{ER}$ a lo largo de la evolución de un paciente, debe considerarse que su comportamiento va a depender principalmente de dos de las determinantes de la $\mathrm{SvO}_{2}$ siempre que los otros se mantengan constantes, de la $\mathrm{DO}_{2}$ dada por el GC y del $\mathrm{VO}_{2}$ dado por el metabolismo celular; entendiendo esto, debemos considerar las siguientes posibilidades al analizar $\mathrm{O}_{2}$ ER (Tabla 1).

\section{Perfusión tisular y $\mathrm{O}_{2} \mathrm{ER}$}

Al contar con una muestra venosa central y una arterial, y aplicar las herramientas mencionadas previamente, se puede realizar un análisis más profundo integrando las variables de perfusión tisular y de $\mathrm{O}_{2} \mathrm{ER}$, mostrado en la figura 1.

\section{Papel del ultrasonido}

Identificadas alteraciones de la perfusión tisular y la $\mathrm{O}_{2} \mathrm{ER}$, el siguiente paso es identificar la alteración del sistema cardiovascular responsable de dichas manifestaciones. El ultrasonido juega un papel fundamental en escenarios de recursos limitados y parte del monitoreo básico ${ }^{12}$.

Existen dos situaciones asociadas con incremento en la mortalidad, a mayor tiempo de duración del choque y mayor cantidad de líquidos administrados mayor riesgo de mortalidad ${ }^{13}$. Existe una variedad de protocolos de evaluación ultrasonográfica para diferentes escenarios 


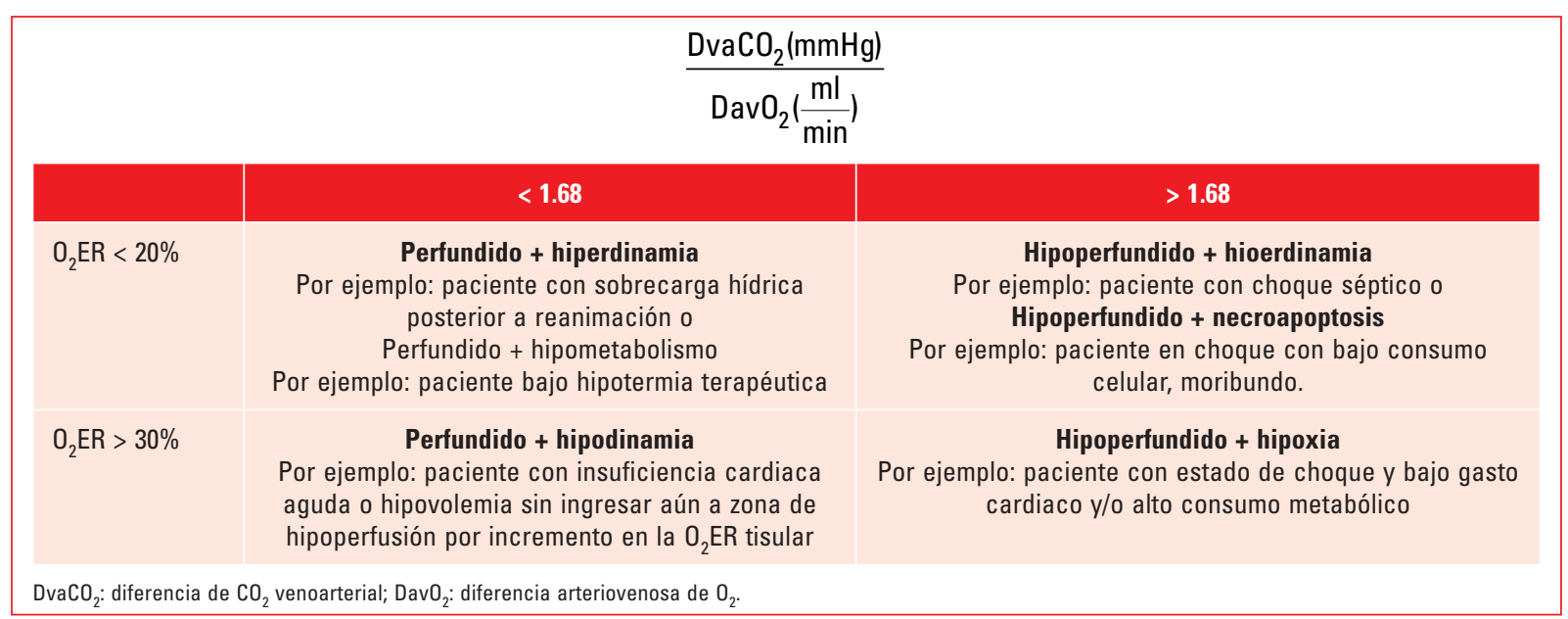

Figura 1. Esquema de las fases y determinantes de saturación venosa central de oxígeno $\left(\mathrm{SvO}_{2}\right)$. Los cambios en cualquiera de los determinantes afecta directamente el valor de $\mathrm{SvO}_{2}$.

clínicos, sin embargo, no es práctico aprenderse cada uno y muchas veces se necesita cierto grado de praxia para completarlos; por el contrario un abordaje sencillo y fácil de integrar puede aplicarse en la mayoría de los escenarios, para ello se puede comparar el sistema cardiovascular con un sistema hidráulico que lleva agua a través de una manguera a una bomba, la cual bombea el agua a través de otra manguera, y el agua viaja y regresa a la bomba. Las causas de mal funcionamiento en el sistema pueden describirse de la siguiente manera:

- Que la bomba no funcione bien, es decir que no tenga fuerza adecuada y puede desbordarse el contenido: se evalúa de manera común la ventana subxifoidea, se valora si la movilidad cardiaca es normal, sincrónica y si las paredes de los ventrículos se acercan y engruesan en la sístole. En caso de choque cardiogénico puede ser que el corazón de manera global se mueva lento, se mueva poco, o pueden identificarse ciertas zonas del corazón que no se mueven igual que el resto, como en un infarto. Si el corazón no se contrae y no bombea bien, puede generar incremento de presión de manera retrógrada y desbordar líquido en el intersticio pulmonar, generando un patrón de congestión pulmonar. Esto mismo puede condicionar la vena cava o yugular que no cambie con los movimientos respiratorios ${ }^{14}$.

- Que la bomba no reciba suficiente agua porque no se llenó adecuadamente la manguera de entrada en caso de que le falte agua al sistema: cuando se evalúa la vena cava inferior o la vena yugular interna se puede inferir que le falta agua al sistema, o que al menos tolera la administración de volumen, cuando estas cambian mucho su diámetro con los movimientos respiratorios. Si se aprecian datos sugestivos de tolerancia y deficiencia de volumen intravascular estamos obligados a buscar fugas en el sistema ${ }^{15}$.

- Que se esté fugando agua en alguna parte del sistema: si el sistema no se está llenando de manera adecuada debemos buscar sitios donde se esté fugando el líquido, para eso debemos buscar en la ventana subxifoidea para derrame pericárdico, buscar derrame pleural, edema alveolo-intersticial o líquido libre en el abdomen ${ }^{16}$.

- Que la bomba no reciba suficiente agua porque algo obstruye la entrada de agua: cuando algo obstruye el sistema, un dato muy importante es que la vena cava inferior o la yugular darán datos de no variación de diámetro, aparecerán como mangueras ingurgitadas y rígidas, como si estuvieran por completo llenas de agua; causas que impiden la bomba se llene: derrame pericárdico que colapsa el ventrículo derecho en un tamponade, neumotórax a tensión que impide el retorno venoso, disfunción aguda de ventrículo derecho secundaria a una tromboembolia pulmonar, o como resultado de ventilación mecánica con presiones o volúmenes inadecuadamente elevados.

Tras la evaluación ultrasonográfica dirigida a evaluar las interrogantes previas, se puede integrar la información obtenida para orientarnos sobre el perfil hemodinámico presente, recordando que el paciente crítico puede presentar más de una sola alteración hemodinámica de manera concomitante (Tabla 2). 
Tabla 2. Evaluación ultrasonográfica para integrar el perfil hemodinámico e identificar el origen de la alteración circulatoria

\begin{tabular}{|l|l|l|l|}
\hline \multirow{2}{*}{ Evaluar } & \multicolumn{3}{|c|}{ Tipo de choque } \\
\cline { 2 - 4 } & Cardiogénico & Hipovolémico & Obstructivo \\
\hline $\begin{array}{l}\text { Bomba alterada } \\
\text { (deforme o contracción deficiente) }\end{array}$ & $\begin{array}{l}\mathrm{X} \\
\text { (p. ej., IAM, contracción deficiente) }\end{array}$ & $\begin{array}{l}\mathrm{X} \\
\text { (deforme, beso ventricular) }\end{array}$ & $\begin{array}{l}\text { X } \\
\text { (p. ej., TEP, deforme) }\end{array}$ \\
\hline $\begin{array}{l}\text { Tolera agua } \\
\text { (colapso de VCl o VY) }\end{array}$ & - & $X$ & - \\
\hline $\begin{array}{l}\text { Fuga } \\
\text { (líquido donde no debe) }\end{array}$ & $\begin{array}{l}\mathrm{X} \\
\text { (patrón B pulmonar) }\end{array}$ & $\mathrm{X}$ & $\mathrm{X}$ \\
\hline $\begin{array}{l}\text { No tolera agua } \\
\text { (no colapso de VCl y VY) }\end{array}$ & $\mathrm{X}$ & (tórax, abdomen) & (tamponade) \\
\hline
\end{tabular}

IAM: infarto agudo del miocardio; TEP: tromboembolia pulmonar; VCl: vena cava inferior; VY: vena yugular interna.

\section{Algoritmo de manejo}

Con base en las herramientas descritas previamente, se propone un algoritmo de manejo para la mayoría de los escenarios de recursos limitados de atención de paciente crítico (Fig. 2). Se basa en la sistematización de las siguientes interrogantes:

1. ¿Este paciente está en choque? Establecer o descartar estado de hipoperfusión: consiste en la integración de las ventanas y el IS, y la confirmación con marcadores bioquímicos $\left(\mathrm{DvaCO}_{2}\right.$, lactato y ratio $\mathrm{DvaCO}_{2} / \mathrm{DavO}_{2}$ ) para establecer si existe o no hipoperfusión sistémica.

2. ¿La presión arterial media (PAM) es adecuada? Una vez establecido el diagnóstico de hipoperfusión tisular es muy importante establecer si la PAM es adecuada o no para el paciente. No existe una PAM estándar, en pacientes que no son hipertensos crónicos se puede considerar normal una PAM entre 60 y $70 \mathrm{mmHg}$, sin embargo, en pacientes con hipertensión crónica la curva de autorregulación vascular se encuentra desplazada a la derecha y por lo tanto valores estandarizados de PAM pueden representar hipotensión y condicionar hipoperfusión, por lo que se recomienda un valor de 80 a $85 \mathrm{mmHg}$ como objetivo ${ }^{17}$. Detectada una PAM inadecuada contribuyente a hipoperfusión, no debe retrasarse el inicio de vasopresor por valorar la respuesta a volumen; se recomienda iniciar vasopresor de forma temprana para lograr una PAM perfusora, y reducir el tiempo de hipoperfusión, para poder continuar con la evaluación hemodinámica. A mayor tiempo de hipoperfusión sostenida, mayor posibilidad de evolucionar a la irreversibilidad. Existe sustento para iniciar vasopresor por vía periférica hasta un lapso de 24 horas, con mínimos efectos adversos, siempre y cuando el catéter venoso periférico esté adecuadamente colocado. El vasopresor no resuelve el problema, pero permite ganar tiempo y evitar la sobrecarga hídrica ${ }^{18,19}$.

3. ¿Cómo está la $\mathrm{O}_{2} \mathrm{ER}$ ? La ausencia de hipoperfusión no descarta una alteración cardiovascular. El análisis de la $\mathrm{SvO}_{2}{ }^{20}$, la $\mathrm{DavO}_{2}$ y la $\mathrm{O}_{2} \mathrm{ER}$ permite obtener información que orienta sobre el comportamiento de la hemodinamia en procesos agudos, o del consumo en casos con mayor tiempo de evolución (Tabla 1).

4. ¿Existe causa mecánica de choque? Es imprescindible descartar las condiciones «mecánicas» que pueden condicionar estado de choque y sesgar el análisis del comportamiento hemodinámico. Estas condiciones son: neumotórax, hemorragia oculta (hemotórax o intrabdominal), tamponade cardiaco y tromboembolia pulmonar. La evaluación ultrasonográfica es una herramienta para la identificación y para guiar el procedimiento resolutivo de varias.

5. ¿Más líquido? Una interrogante es si el paciente mejorará tras la administración de volumen, es decir, si el GC mejorará tras optimizar la precarga. Evaluar si administrar volumen puede llegar a ser beneficioso, sobre todo en paciente con alteración renal que no es capaz de eliminar el volumen efectivamente. Cuando un paciente no tiene datos clínicos de sobrecarga hídrica como ingurgitación yugular, datos de congestión pulmonar, acompañada de una presión venosa central $(P V C)<5 \mathrm{mmHg}$, así como una pobre tolerancia hemodinámica a presiones del ventilador cuando se encuentra bajo ventilación mecánica y se incrementa el presión positiva al final de la espiración (PEEP) a $15 \mathrm{cmH}_{2} \mathrm{O}$ (maniobra de potencial de reclutamiento pulmonar), manifestando hipotensión e 


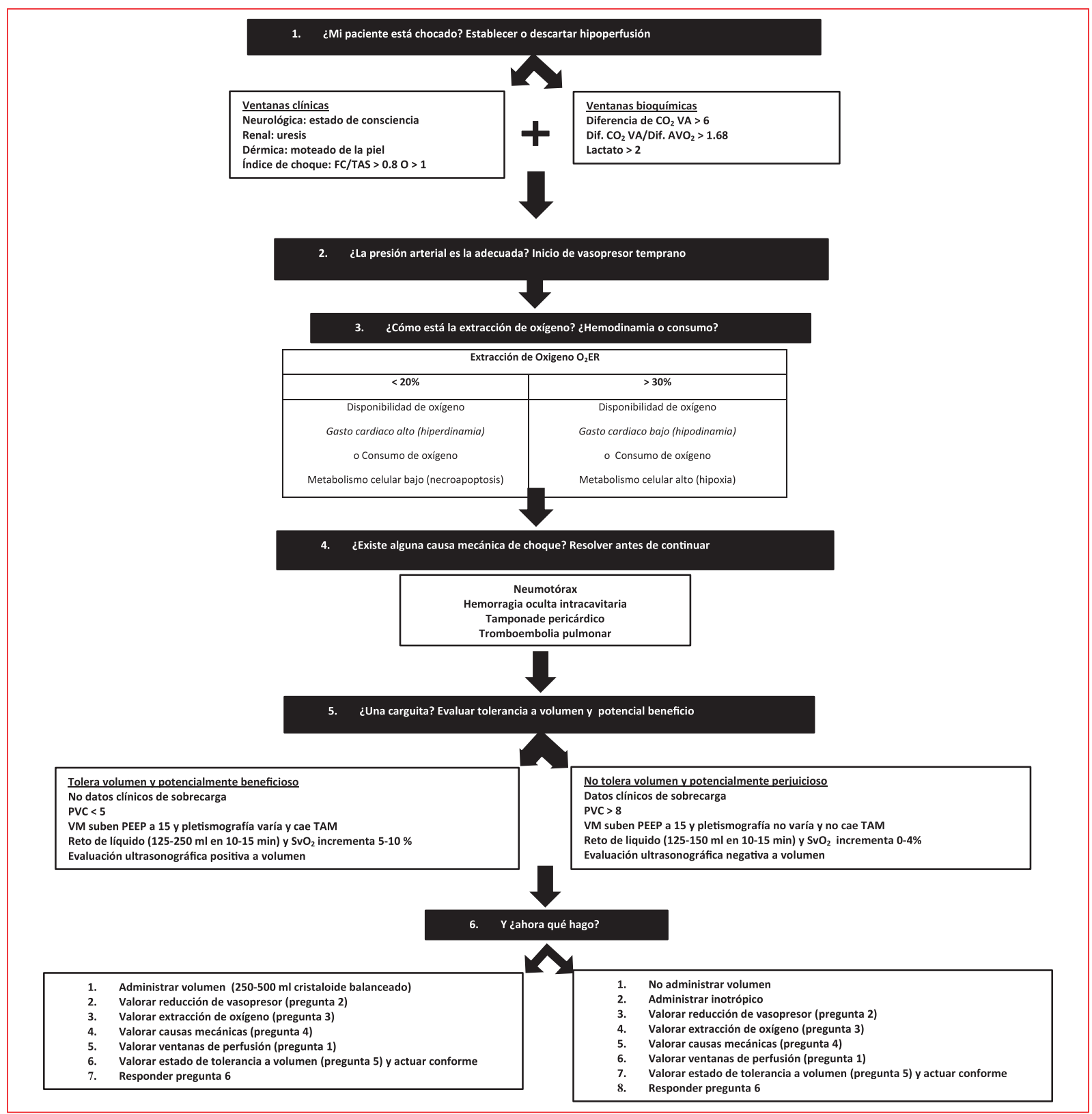

Figura 2. Algoritmo integrador de evaluación y manejo hemodinámico en escenarios con recursos limitados. 02ER: extracción de oxígeno; C02: dióxido de carbono; VA: veno-arterial; AV02: arterio-venosa de oxígeno; FC: frecuencia cardíaca; TAS: tensión arterial asistólica; PVC: presión venosa central; VM: volumen minuto; PEEP: presión positiva al final de la espiración; TAM: presión arterial media.

incremento en la variabilidad de la onda de pletismografía ${ }^{21}$; también cuando se realiza un reto de líquido con 100-150 ml máximo de solución cristaloide o elevación pasiva de las piernas al menos 2 minutos, el registrar un incremento en el valor de la $\mathrm{SvO}_{2}$ de $5-10 \%$ es sugestivo de tolerancia y de beneficio potencial al administrar volumen ${ }^{22}$. Cuando el paciente cuenta con todo lo anterior contrario, es decir datos de sobrecarga, PVC > $8 \mathrm{mmHg}$, el incrementar el PEEP a 15 no genera ninguna alteración hemodinámica 0 en la onda de pletismografía, y al realizar elevación pasiva de las piernas o realizar un reto de líquido no se genera un incremento mayor a $4 \%$ en el valor de $\mathrm{SvO}_{2}$. En caso de contar con la posibilidad de realizar una evaluación ultrasonográfica se recomienda realizar el análisis propuesto previamente 
para integrar el perfil hemodinámico y por tanto la tolerancia o no del sistema y su potencial beneficio o perjuicio a la administración de volumen.

6. ¿Y ahora qué hago? Intervenir y revaluar:

a. En caso de cumplir con datos de tolerancia a volumen y un potencial beneficio en su administración: administrar una cantidad de cristaloide, de preferencia soluciones balanceadas, como la solución Hartmann, que tienen menor contenido de cloro con respecto a la solución salina al $0.9 \%$, en una cantidad de $250 \mathrm{ml}$ preferentemente y como máximo $500 \mathrm{ml}$ en un lapso de 15-20 minutos, y valorar la optimización del sistema cardiovascular mediante reducción en la dosis de vasopresor necesaria para mantener presión arterial media (TAM) perfusora, acompañado de una mejoría en el comportamiento de las variables que reflejan comportamiento del GC y finalmente lo más importante, en mejoría de las variables que reflejan hipoperfusión tisular.

b. En caso de cumplir con datos de intolerancia a volumen y un potencial perjuicio en su administración: no administrar más volumen, el inicio de inotrópico (levosimendán, dobutamina, epinefrina) es una opción viable y fisiológicamente respaldada por el análisis desarrollado previamente, donde el volumen sistólico no mejorará a la administración de volumen. Tras la administración del inotrópico se debe valorar la optimización del sistema cardiovascular mediante: reducción en la dosis de vasopresor necesaria para mantener TAM perfusora, acompañado de una mejoría en el comportamiento de las variables que reflejan comportamiento del GC y finalmente lo más importante, en mejoría de las variables que reflejan hipoperfusión tisular. Tras lo anterior, al cabo de 15-30 minutos se sugiere repetir la evaluación (la evaluación de la perfusión tisular de manera bioquímica puede ser cada 30 minutos a 1 hora hasta lograr su restauración, y posteriormente con intervalos de 2-4 horas). Recordar que con las intervenciones realizadas un sistema tolerante puede llegar a un punto de intolerancia y viceversa.

\section{Conclusiones}

Existen diversas herramientas de fácil acceso y disponibilidad, basadas en principios fisiológicos y fisiopatológicos que brindan información para la evaluación y manejo hemodinámico y permiten apegarse a los principios de seguridad e individualización, aplicables en nuestra realidad hospitalaria. La integración de las variables descritas en el presente capítulo resalta la factibilidad de brindar atención médica eficaz y eficiente al optimizar los recursos que han estado en su mayoría disponibles a nuestro alcance desde hace tiempo y que hoy obtienen un peso y un valor agregado en comparación al análisis aislado de cada una de ellas. Finalmente, el paciente críticamente enfermo tiene la mejor posibilidad de sobrevivir cuando recibe el tratamiento que necesita a la mayor brevedad posible independientemente del escenario donde se encuentre.

\section{Bibliografía}

1. Marshall JC, Bosco L, Adhikari NK, Connolly B, Diaz JV, Dorman T, et al. What is an intensive care unit? A report of the task force of the World Federation of Societies of Intensive and Critical Care Medicine. Crit Care. 2017;37:270-6.

2. Cecconi M, De Backer D, Antonelli M, Beale R, Bakker J, Hofer C, et al. Consensus on circulatory shock and hemodynamic monitoring. Task force of the European Society of Intensive Care Medicine. Intensive Care Med. 2014;40(12):1795-815.

3. Vincent JL, Ince C, Bakker J. Clinical review: Circulatory shock-an update: a tribute to Professor Max Harry Weil. Crit Care. 2012;16(6):239.

4. Campos-Serra A, Montmany-Vioque $S$, Rebasa-Cladera $P$, Llaquet-Bayo H, Gàrcia-Roman R, Colom-Gordillo $A$, et al. The use of the Shock Index as a predictor of active bleeding in trauma patients. Cir Esp. 2018;96(8):494-500.

5. Kuster M, Exadaktylos A, Schnüriger B. Non-invasive hemodynamic monitoring in trauma patients. World J Emerg Surg. 2015;10:1116.

6. Mallat J, Lemyze M, Tronchon L, Vallet B, Thevenin D. Use of venous-to-arterial carbon dioxide tension difference to guide resuscitation therapy in septic shock. World J Crit Care Med. 2016;5(1):47-56.

7. Dubin $\mathrm{A}, \mathrm{Pozo} \mathrm{MO}$. Shedding light on venoarterial $\mathrm{PCO}_{2}$ gradient. Ann Intensive Care. 2017;7(1):41.

8. Ospina-Tascón GA, Hernández G, Ceconni M. Understanding the venous-arterial $\mathrm{CO} 2$ to arterial-venous $\mathrm{O} 2$ content difference ratio. Intensive Care Med. 2016;42(11):1801-4.

9. Mallat J, Lemyze M, Meddour M, Pepy F, Gasan G, Barrailler S, et al. Ratios of central venous-to-arterial carbon dioxide content or tension to arteriovenous oxygen content are better markers of global anaerobic metabolism than lactate in septic shock patients. Ann Intensive Care. 2016;6:10.

10. Gattinoni L, Pasenti A, Matthay M. Understanding blood gas analysis. Intensive Care Med. 2017 doi: 10.1007/s00134-017-4824-y.

11. Molnar Z, Nemeth M. Monitoring of tissue oxygenation: an everyday clinical challenge. Front Med (Lausanne). 2018;4:247.

12. Chacko J, Brar G. Bedside ultrasonography: Applications in critical care: Part I. Indian J Crit Care Med. 2014;18(5):301-9.

13. Acheampong $\mathrm{A}$, Vincent $\mathrm{JL}$. A positive fluid balance is an independent prognostic factor in patients with sepsis. Crit Care. 2015;19:251.

14. Blaivas M. Incidence of pericardial effusion in patients presenting to the emergency department with unexplained dyspnea. Academic Emerg Med. 2001;8(12):1143-6.

15. Seif D, Perera, Mailhot T, Riley D, Mandavia D. Bedside ultrasound in resuscitation and the rapid ultrasound in shock protocol. Crit Care Research Pract. 2012;2012:503254.

16. Perera P, Mailhot T, Riley D, Mandavia D. The RUSH exam: rapid ultrasound in shock in the evaluation of teh critically ill. Emerg Med Clin N Am. 2010;28:29-56.

17. Hylands M, Toma A, Beaudoin N, Frenette AJ, D'Aragon F, Belley-Côte É, et al. Early vasopressor use following traumatic injury: a systematic review. BMJ Open. 2017;7(11):e017559.

18. Peake LS, Delaney D, Bailey AM, Bellomo R, Cameron PA, Cooper DJ, et al. Goal-directed resuscitation for patients with early septic shock. N Engl J Med. 2014;371:1496-506.

19. The ProCESS Investigators. A randomized trial of protocol-based care for early septic shock. N Engl J Med. 2014;370:1683-93.

20. Byrne AL, Bennett M, Chatteriji R, Symons R, Pace NL, Tomas PS. Peripheral venous and arterial blood gas analysis in adults: are they comparable? A systematic review and meta-analysis. Respirology. 2014;19(2):168.

21. Marik PE, Cavallazzi R, Vasu T, Hirani A. Dynamic changes in arterial waveform derived variables and fluid responsiveness in mechanically ventilated patients: a systematic review of the literature. Crit Care Med. 2009;37:2642-7

22. van Beest $P$, Wietasch $G$, Scheeren T, Spronk P, Kyuiuper M. Clinical review: use of venous oxygen saturations as a goal - a yet unfinished puzzle. Crit Care. 2011;15(5):232. 